\title{
Cyclical vomiting syndrome in a 10 year old girl: a case report
}

\author{
Swarna Wijetunga, Manisha Dalpatadu
}

\section{Summary}

A 10 year old girl presented with repeated episodes of vomiting over a one year period. She had intense nausea and severe vomiting, occurring in cycles which needed repeated hospital admissions, severely affecting her functioning. Management was instituted in four phases and she responded well to her treatment.

SL J Psychiatry 2011; 2 (2):70-72

\section{Introduction}

The occurrence of cyclical vomiting syndrome (CVS) was first described by Heberden in 1806 in France and by Gee in 1882 in Britain (1). Previously it was thought that CVS was an entity occurring mostly in children. Physicians attempting to identify a medical cause for vomiting, when confronted with negative results after extensive investigations assumed the cause was psychological. This disorder is characterized by severe nausea and vomiting lasting several days, recurring several times per year with symptom free intervals in between episodes. The onset may be at anytime from childhood to adulthood.

CVS is frequently accompanied by anxiety, panic attacks and depression in adults as well as in children. Recent research suggests a variety of possible etiologies, including mitochondrial enzymopathies, cannabinoid hyperemesis, gastrointestinal dysmotility, Corticotropin Releasing Factor (CRF)-Adrenocorticotpic hormone $(\mathrm{ACTH})$ dysfunction and autonomic dysfunction. However it is known that psychosocial factors also can act as triggers for CVS. (1)

The exact etiology and pathogenesis of CVS is still unknown but an integrated management approach which combines pharmacological, psychotherapeutic, psycho-educational and lifestyle modification has resulted in good outcomes and a better quality of life(2).

\section{Case report}

A ten year old school girl from southern Sri Lanka presented to the Child and Adolescent Psychiatry Clinic with recurrent, severe episodes of vomiting over a period of one year.

After successfully completing a year five scholarship examination, the patient entered a new school. After attending this school for three days, she developed a viral fever. The fever resolved on the sixth day, but thereafter she had repeated episodes of severe vomiting at school. Her vomiting was preceded by bilateral headache, generalized body aches and excessive sleepiness. This phase usually occurred between 4 a.m. and 7 a.m. Following this phase, she developed severe nausea and vomiting. The initial vomitus consisted of food particles; later it contained bile and was followed by white froth.

After the patient begins to vomit she has severe retching, repeatedly consumes water and vomits all the fluid consumed, again. Episodes of drinking water and vomiting occurs throughout the day. Initially she experienced about six episodes per month. Thereafter the cycles of vomiting lasted about one week with severe vomiting lasting for about two days. Once the vomiting phase resolved, the patient resumed normal eating within two days. Thereafter, until her next bout of vomiting she reverted to her usual level of functioning. The patient was extensively investigated and treated by pediatricians and by neurologists to exclude a neurological cause for recurrent vomiting. All routine investigations including a Magnetic Resonance Imaging (MRI) scan of the brain were normal. She was then referred to a psychiatrist to exclude a psychological problem associated with vomiting.

An extensive evaluation was carried out by the Child Psychiatry team. The patient had a normal developmental history. Her past medical history was unremarkable. There was no past history or family history suggestive of migraine. She was anxious and distressed but was not clinically depressed. There were no features suggestive of panic attacks.

The patient identified as recent stressors her school tests and the remarks of a friend who had irritated her by saying she is not capable of performing well in studies. Her perpetuating stress factor was the ongoing illness which interfered with her school attendance. The behavior of her parents when the child was ill was identified as a reinforcing factor. Her temperament was that of a high achiever. She had perfectionist traits. She was overly concerned and fearful of failure.

On physical examination the patient looked emaciated. Neurological examination was normal. 


\section{Management}

The patient was admitted to the in-patient child psychiatry unit at a tertiary care children's' hospital. She was observed for vomiting, factors which triggered vomiting and psychosocial factors affecting the patient and the family were explored.

She was moderately dehydrated during the initial days of her admission to hospital; this was corrected with intra-venous fluids. Anti-emetics were also administered. Once her physical condition was stable a formal assessment was carried out. Triggers for vomiting were identified as a spicy preparation of fish, stressors such a fear of upcoming examinations and conflicts with, peers at school.

Her management focused on four main aspects:

1. Management of the prodromal phase: $A$ combination of relaxation techniques and distraction methods were used in conjunction with with antiemetics and anxiolytics.

2. Management of the vomiting phase: Supportive management with attention to hydration and electrolyte imbalances was employed.

3. Management during the inter-episodic period: Cognitive behaviour therapy (CBT) was commenced. Along with $\mathrm{CBT}$, problem solving techniques were taught and supportive therapy was provided. The family was supported with education and information on how best to approach such vomiting episodes without reinforcing such behaviour. The Parents were educated on better parenting techniques. Star charts were used to reward good behavior. The patient was instructed to maintain a vomiting diary.

4. Life style modification included the following: Avoiding sleep deprivation, extremely stressful situations and triggering foods were encouraged.

\section{Discussion}

Cyclical vomiting syndrome (CVS) is characterized by severe nausea and vomiting lasting for several days, recurring several times per year, with symptom free intervals in between episodes. CVS is typically characterized by four phases: prodromal phase, vomiting phase, recovery phase and the interepisodic phase $(4,5)$. New criteria for diagnosing children with CVS have been suggested in the North American Society for Pediatric Gastroenterology, Hepatology, and Nutrition Consensus Statement (3).

During the prodromal phase our patient experienced headache, generalized body aches and excessive sleepiness. Identification of the symptoms during the prodromal phase is important to arrive at a diagnosis. Headache and photophobia is suggestive of a migraine. Abnormal eye movements and focal neurological signs indicate a possible neuropathological etiology. Severe abdominal pain is indicative of an intermittent volvulus presenting as episodic vomiting.
Our patient's headache was mainly bilateral and also involved the frontal area. There was no preceding aura. During this phase an antiemetic, an anxiolytic as well as a sedative were prescribed as abortive therapy to prevent the patient from lapsing into the vomiting phase.

During the vomiting phase intense nausea and vomiting was observed in our patient. In this phase the main focus was on the general management of the patient with monitoring of biochemical parameters and adequate hydration when needed. Treatment of CVS must be prompt. If delayed, the patient's extreme distress may precipitate another episode, and since anticipatory anxiety can cause nausea, this may cause more frequent episodes and a coalescent pattern of attacks. Episodes that cannot be aborted should be treated without delay, ideally within an hour of onset.

One sign observed during the vomiting phase in our patient was repeated drinking of water which was regurgitated. Many patients experience intense thirst that compels them to drink, even though they know that the ingested water will be vomited out almost immediately. Drinking dilutes acid and bile, thereby making the vomitus less of a contact irritant to the esophagus and mouth. Emesis can be induced more easily from a full stomach than from an empty stomach. The transient lessening of nausea that follows self-induced vomiting makes this a comfort-seeking behavior in some patients. Preventing the patient from drinking water at all during the vomiting phase is not possible. Sedation is a measure adopted to eliminate this drink-and-vomit cycle.

The recovery phase begins when vomiting and retching ceases and the nausea subsides. It ends when the patient's appetite, tolerance for food and vigor return to normal. The vomiting phase lasted five to six days in our patient and other symptoms also subsided after about two days.

During the inter-episodic phase of CVS patient resume normal eating. There are no further episodes of vomiting or nausea. In this phase our patient was taught how to engage in relaxation therapy with breathing exercises which could be carried out during the prodromal phase of vomiting.

Problem solving techniques to address her anxiety regarding facing examinations were taught to the patient and advice was provided on coping skills especially with regard to peer issues.

General management strategies included reassurance that episodes are not self-induced and education regarding illness, maintaining a 'vomiting diary' for potential precipitating factors and recognising the potential role of excitement as a trigger.

Lifestyle modifications that were attempted included avoiding fasting, maintaining good sleep hygiene and avoiding triggering foods such as chocolate, cheese and monosodium glutamate. 
Supplemental carbohydrates were used in the management of fasting-induced episodes and included fruit juices, other sugar-containing drinks and extra snacks between meals, before exertion, or at bedtime. Lifestyle interventions were attempted to prevent migraine attacks and included regular aerobic exercise (while avoiding over exercising) and regular meal schedules (while avoiding skipping meals). Moderation or avoidance of caffeine was also attempted.

These measures were found to be helpful in preventing further attacks during this patient's stay in hospital.

There is little data about the long-term outcome in CVS. In a study by Fleisher et al of 41 adults with CVS with an age of onset ranging from 2-49 years, after a follow-up averag $\rightarrow$ ing 3.8 years, $86 \%$ reported feeling better after intervention, with fewer attacks and less time spent feeling sick(6).

CVS is a potentially incapacitating disorder affecting all ages, first recognized in children but increasingly documented in adults as well. The prevalence and pathophysiology are currently unknown.

Some patients with CVS have increased psychiatric comorbidity with anxiety or depression being common presentations. They improve with psychiatric treatment. Integrated treat $\neg$ ment combining pharmacological, psychotherapeutic, psycho $\neg$ educational and lifestyle aspects as well as family therapy provides the best chances for successful treatment.

\section{Declaration of interest}

None

Swarna Wijetunga, Consultant Psychiatrist, Child and Adolescent Mental Health Services Lady Ridgeway Childrens Hospital, Colombo, Sri Lanka

Manisha Dalpatadu, Registrar, Child and

Adolescent Mental Health Services Lady

Ridgeway Childrens Hospital, Colombo, Sri Lanka

Corresponding author

Manisha Dalpatadu, Registrar, Lady Ridgeway

Childrens Hospital, Colombo, Sri Lanka

E mail: manisha_dalpatadu@yahoo.com

\section{References}

1. Sonje S, Levenson JL. Cyclic vomiting syndrome, part 1. Primary Psychiatry. 2009;16(6):15-18.

2. Sonje S, Levenson JL. Cyclic vomiting syndrome, part2 Primary Psychiatry 2009;16(9):25-28

3. Li BU, Lefevre F, Chelimsky GG, Boles RG, Nelson SP, Lewis DW, Linder SL, Issenman RM, Rudolph CD; North American Society for Pediatric Gastroenterology, Hepatology, and Nutrition.North American Society for Pediatric Gastroenterology, Hepatology, and Nutrition Consensus Statement on the Diagnosis and Management of Cyclic Vomiting Syndrome -Journal of Pediatric Gastroenterology and Nutrition 200847:379-393
4. Fleisher DR Empiric guidelines for treatment of cyclic vomiting syndrome April, 2008 http://www.cvsa.org.uk/ downloads/Fleisherguidlines.pdf

5. Fleisher DR, Gornowicz B, Adams K, Burch R, Feldman EJ. Cyclic Vomiting Syndrome in 41 adults: the illness, the patients, and problems of management. BMC Med. 2005;3:20. CrossRef 